Energieunterschied $X$ in erster Năherung konstant (gleichbleibende $\mathrm{C}_{2 \mathrm{p}}-\mathrm{L}_{2 \mathrm{p}}$-Überlappung). Mit wachsender LUMOEnergie des Carbens ${ }^{[3]}$ nimmt daher die elektrophile Reaktivität ab und die nucleophile zu. $\mathrm{C}\left(\mathrm{OCH}_{3}\right)_{2}$ reagiert im $\mathrm{Ge}$ gensatz zum $\mathrm{CF}_{2}$ nucleophil ${ }^{[8]}$. Gleichzeitig nimmt die Selektivität nucleophiler Carbene in der Liganden-Reihenfolge $\mathrm{L}=\mathrm{OCH}_{3}<\mathrm{N}\left(\mathrm{CH}_{3}\right)_{2} \mathrm{zu}^{[8]}$.

Die Untersuchungen zeigen also, daß sich auf der Grundlage einer differentiellen Formulierung ${ }^{(9)}$ des Grenzorbital-Modells die Carbene in zwei Klassen einteilen lassen, deren Reaktivităts-Selektivitäts-Beziehung entweder einem direkten oder einem inversen Zusammenhang gehorcht. Dieses für Carbene abgeleitete Konzept gilt allgemein für Grenzorbital-kontrollierte Reaktionen, z. B. Cycloadditionen und Radikalreaktionen ${ }^{[?]}$.

Eingegangen am 17. Februar 1981 , in geănderter Fassung am 23. Mărz 1981 [Z 811]

[1] W. Kirmse: Carbene Chemistry, Academic Press, New York 1971; R. A. Moss in M. Jones, Jr.: Carbenes, Vol. I, Wiley, New York 1973.

[2] B. Giese, Angew. Chem. 89, 162 (1977); Angew. Chem. Int. Ed. Engl. 16, 125 (1977).

[3] $W$. W. Schoeller. Tetrahedron Lett. 1980, 1505; siehe auch W. W. Schoeller, U. H. Brinker, Z. Naturforsch. B 35, 475 (1980)

[4] E. Heilbronner, H. Bock: Das HMO-Modell und seine Anwendung, Verlag Chemie, Weinheim 1968.

[5] Die Änderung der Grenzorbitalenergien im Olefin ist klein gegenuber den absoluten HOMO-LUMO-Differenzen. Dies laßit sich aus den experimentell bestimmten Orbitalenergien abschătzen (siehe auch [6]).

[6] Abgeschătzt nach J. S. Shapiro, F. P. Lassing. J. Phys. Chem. 72, 1552 (1968); D. E. Milligan, M. E. Jacox, J. Chem. Phys. 47, 703 (1967), zit. Lit. Im Vergleich mit $\mathrm{CF}_{2}$ wird in $\mathrm{CCl}_{2}$ das HOMO stärker angehoben als das LUMO gesenkt.

(7) Berechnet nach der HMO-Theorie unter Verwendung von Standardparametern fü die Heteroatome. A. Streitwieser, $J r$. Molecular Orbital Theory for Organic Chemists, Wiley, New York 1961.

[8] a) R. W. Hoffmann. B. Hagenbruch, D. M. Smith, Chem. Ber. 110, 23 (1977); K. Steinbach, Dissertation, Universităt Marburg 1974. Professor R. W. Hoffmann danke ich für den Hinweis auf diese Ergebnisse; b) MNDO-Rechnungen stimmen mit diesem Sachverhalt überein. For die Reaktion der Carbene ( $\sigma^{2}$-Konfiguration) $\mathrm{CF}_{2}, \mathrm{C}(\mathrm{OH})_{2}, \mathrm{C}\left(\mathrm{NH}_{2}\right)_{2}$ und $\mathrm{CCl}_{2}$ mit den Substraten Ethylen (1,1-Dihydroxyethylen, 1,1-Difluorethy. len) ergeben sich die Energiebarrieren (in kcal/mol für die total bestimmten Energiehyperflachen) $21.9(17.4,21.7) ; 24.5(28.6,21.0) ; 21.4(27.2$ 14.6); $12.5(8.2,15.8)$

[9] FaBt man $E$ in Gi. (a) als totales Differential auf, so läBt sich eine allgemeingültige Reaktivităts-Selektivităts-Beziehung herleiten; $\boldsymbol{W}$. $\boldsymbol{W}$. Schoeller, noch unveröffentlichte Ergebnisse.

\section{Olefinadditionen an}

\section{2,5-Diphenyl-1,3,4-oxadiazin-6-on ${ }^{[* "]}$}

Von Manfred Christl, Ulrike Lanzendörfer und Silke Freund $\left.{ }^{*}\right)$

\section{Professor Siegfried Hünig zum 60. Geburtstag gewidmet}

1977 stellten Steglich et al. ${ }^{[1]}$ erstmals 2,5-Diphenyl-1,3,4oxadiazin-6-on (1) her und setzten es mit 1-Diethylaminopropin und mit Dehydrobenzol um. Dabei folgt der DielsAlder-Addition eine Cycloreversion unter Stickstoffabspaltung und $\alpha$-Pyronbildung. Wir berichten über Reaktionen von Olefinen mit (l), die es ermőglichen, gleichzeitig eine Benzoyl- und eine Phenylketenylgruppe an eine CCDoppelbindung zu addieren.

In etherischem Benzvalen (2), einem Olefin hoher Aktivität in Diels-Alder-Additionen mit inversem Elektronen-

[*] Prof. Dr. M. Christl, Dipl.-Chem. U. Lanzendorfer, Dipl.-Chem. S. Freund Institut für Organische Chemie der Universităt Am Hubland, D-8700 Würzburg

[**] Diese Arbeit wurde von der Deutschen Forschungsgemeinschaft und dem Fonds der Chemischen Industrie unterstützt. bedarf ${ }^{2 !}$, wandelt sich suspendiertes (1) bei $20^{\circ} \mathrm{C}$ innerhalb von $24 \mathrm{~h}$ in das mit $75 \%$ Ausbeute isolierte tetracyclische Diketon (6) um. Wahrscheinlich bildet das Primäraddukt (3) in einer $\left[\sigma^{2}+{ }_{\sigma} 2+{ }_{\sigma} 2\right]$-Cycloreversion unter Stickstoffabspaltung und Lösung der Lactonbindung (4-Benzoyltricyclo[3.1.0.0 ${ }^{2,6}$ ]hex-3-yl)phenylketen (4). In einer neuartigen Cycloaddition lagert sich dann die elektrophile Ketenfunktion intramolekular an das nucleophile Bicy. clo[1.1.0]butan-System, woraus schließlich der Tetracyclus (6) resultiert, möglicherweise über das Zwitterion (5). Für die Strukturzuordnung von (6) ist das ${ }^{13} \mathrm{C}-\mathrm{NMR}$-Spektrum (Tabelle 1) entscheidend: Die direkten ${ }^{13} \mathrm{C}$-H-Kopplungskonstanten zeigen, daß fünf der sechs $\mathrm{CH}$-Gruppen Cyclopropaneinheiten angehören, die sechste aber einem Cyclopentan.
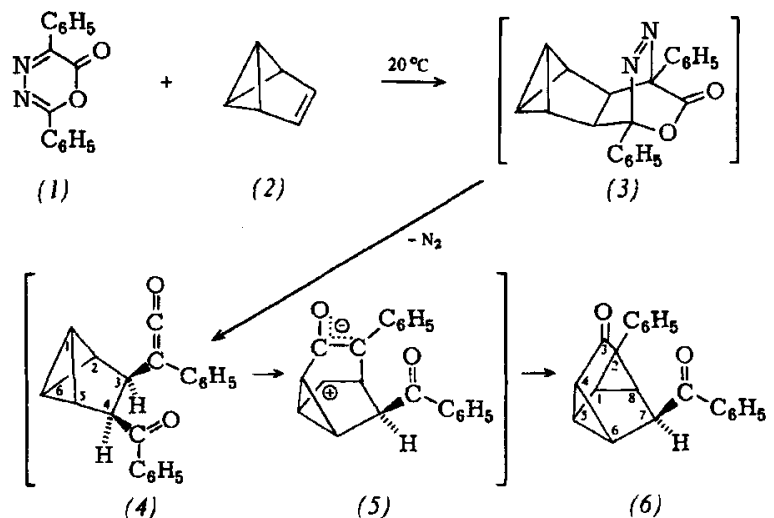

Tabelle 1. Einige physikalische Daten der neuen Verbindungen [NMR-Spektren in $\mathrm{CDCl}_{3}$, bei (9) $\left(\mathrm{CF}_{3}\right)_{2} \mathrm{CO} \cdot 1.5 \mathrm{D}_{2} \mathrm{O}, \delta$-Werte; IR-Spektren in $\mathrm{KBr}$.

(6), $\mathrm{Fp}=140-142^{\circ} \mathrm{C}$; IR: $1700(3-\mathrm{C}-0), 1670 \mathrm{~cm}^{-1}$ (Benzoyl-C-O): 'HNMR: $1.93\left(\mathrm{dd}, 4-\mathrm{H}, J_{4,5}=5.7 \mathrm{~Hz}, J_{4,5}=9.0 \mathrm{~Hz}\right), 2.1-2.4(\mathrm{~m}, 6-\mathrm{H}, 8-\mathrm{H}), 2.61$ (dt, 5-H, $J_{5.6}=J_{1,5}=5.1 \mathrm{~Hz}$ ), $2.88\left(\mathrm{dd}, 1-\mathrm{H}, J_{1,8}=5.7 \mathrm{~Hz}\right), 4.58($ t, $7 \cdot \mathrm{H}$, $J_{0,7}=J_{0}=4.1 \mathrm{~Hz}$, $7.30\left(\mathrm{~s}, 2-\mathrm{C}_{6} \mathrm{H}_{3}\right), 73-7.7$ und 7.9-8.2 (jeweils $\mathrm{m}, 3 \mathrm{H}$ und $2 \mathrm{H}$, Benzoyl- $\mathrm{C}_{6} \mathrm{H}_{5}$ ); ${ }^{13} \mathrm{C}-\mathrm{NMR}: 25.9,30.2,32.6,34.1,40.6$ (jeweils d, 185, $179,183,176,173 \mathrm{~Hz}, \mathrm{C}-1, \mathrm{C}-4, \mathrm{C}-5, \mathrm{C}-6, \mathrm{C}-8), 43.7$ (s, C-2), 46.7 (d, $131 \mathrm{~Hz}$, C-7), 198.1, 204.9 (jeweils s, C-3, Benzoyl-CO), $\mathrm{C}_{6} \mathrm{H}_{5}: 127.2,133.3$ (jeweils d, C-4, C-4'), 128.3 (Doppelbande), 128.8, 129.7 (jeweils d, C-2, C-3, C-2', C-3'), 135.3, 136.9 (jeweils $s, C-1, C-1^{\prime}$ )

(9). $\mathrm{Fp}=201-203^{\circ} \mathrm{C}$; IR: $1755 \mathrm{~cm}^{-1}(\mathrm{C}-\mathrm{O})$; 'H-NMR: 0.39 (br. d, 7-H $J_{7.7} \approx 10 \mathrm{~Hz}$ ), 1.00 (br. d, 7- $\left.\mathrm{H}_{a n t i}\right), 1.5-2.0$ (m, $\left.2 \times 5-\mathrm{H}, 2 \times 6-\mathrm{H}\right), 2.44$ (br. s, 4 . H), 2.97 (br. d, 3-H, $J_{3.8}=7.0 \mathrm{~Hz}$ ), 3.00 (br. s, 1-H), 4.28 (d, 8-H), 7.0-7.8 (m, $2 \mathrm{C}_{6} \mathrm{H}_{5}$ )

(10), $\mathrm{Fp}=210^{\circ} \mathrm{C}$; IR: $1750 \mathrm{~cm}^{-1}(\mathrm{C}=0)$

(11) $\mathrm{Fp}=153-155^{\circ} \mathrm{C}$; IR: 1740 (Ester-C-0), $1674 \mathrm{~cm}^{-1}$ (Benzoyl-C-O); 'H-NMR: $1.58\left(\mathrm{dq}, 7 . \mathrm{H}_{\text {anti }}, J_{7.7}=8.7 \mathrm{~Hz}, J_{1,7 \text { andi }} \approx J_{3.7 \text { anit }} \approx J_{4,7 \text { anti }} \approx 1.8 \mathrm{~Hz}\right.$ ), 1.78 (br. d, 7-H $\mathrm{H}_{3, \mathrm{~m}}$ ), 2.77 (m, 4-H), $3.07(\mathrm{~m}, 1-\mathrm{H}), 3.10$ (ddd, $3-\mathrm{H}, J_{3,8}=11.4 \mathrm{~Hz}$, $J_{2,3}=4.5 \mathrm{~Hz}$ ), $3.33\left(\mathrm{dd}, 2-\mathrm{H}, J_{1.2}=3.3 \mathrm{~Hz}\right), 3.50(\mathrm{~d}, 8-\mathrm{H}), 3.67\left(\mathrm{~s}, \mathrm{OCH}_{3}\right), 5.72$ (dd, $\left.5 \cdot \mathrm{H}, J_{5,6}=5.4 \mathrm{~Hz}, J_{4,5}=3.0 \mathrm{~Hz}\right), 6.45\left(\mathrm{dd}, 6-\mathrm{H}, J_{1,6}=3.0 \mathrm{~Hz}\right), 6.9-7.6(\mathrm{~m}$, $\left.2 \mathrm{C}_{6} \mathrm{H}_{5}\right)$

(12). $\mathrm{Fp}=195-196^{\circ} \mathrm{C} ;$ IR: $1754 \mathrm{~cm}^{-1}(\mathrm{C}-0)$

Norbornen (7) reagiert erst in siedendem Tetrachlormethan mit (1). Im Gegensatz zur Addition von (2) ist hier die Ketenzwischenstufe mit Benzoylfunktion (8) intermediär durch IR-Banden der Lösung bei 2100 und $1680 \mathrm{~cm}^{-1}$ nachweisbar, jedoch nach $10 \mathrm{~h}$ Reaktionszeit wieder vollständig verschwunden. Dann isoliert man mit 35\% Ausbeute das Enollacton (9), das vermutlich durch Enolisierung und intramolekulare Addition der Enolhydroxygruppe an die Ketenfunktion aus (8) hervorgeht. Dieser 
langsame Prozeß kommt zum Zuge, weil das überbrückte Cyclopentan in (8) weit weniger nucleophil ist als das Bicyclo[1.1.0]butan in (4).<smiles>N#CC1=CC2CCC1C2</smiles>

(7)

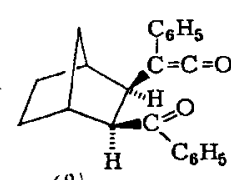

(8)

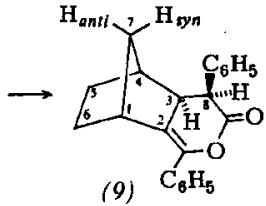

(9) $\mathrm{C}_{8} \mathrm{H}_{5}$

Das 'H-NMR-Spektrum von (9) (Tabelle 1) liefert Argumente für die Konfiguration an C-3 und C-8. Weil $J_{3.4}$ nicht aufgelost ist, sollte 3-H endo-ständig sein, was den erwarteten exo-Angriff von (1) auf (7) anzeigt. Die große Differenz zwischen den chemischen Verschiebungen der beiden 7-H-Atome rührt daher, daß 7- $\mathrm{H}_{\text {syn }}$ in den Abschirmungsbereich der 8-Phenylgruppe gelangt, womit deren exo-Anordnung naheliegt.

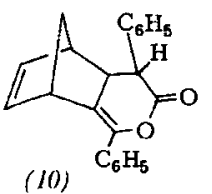

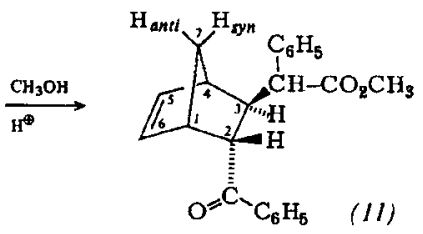

Aus (1) und Norbornadien in Tetrachlormethan ist nach $4 \mathrm{~d}$ bei $45-50^{\circ} \mathrm{C}(10)$ mit $43 \%$ Ausbeute erhältlich. Seine Umsetzung mit Methanol unter Schwefelsăurekatalyse führt zur Öffnung des Lactonrings. In (11) (73\% Ausbeute) zeigen die Kopplungskonstanten von $2-\mathrm{H}$ und $3-\mathrm{H}$ im ' $\mathrm{H}$ NMR-Spektrum, daß die Benzoylfunktion die endo- und die Phenylessigsäureestergruppe die exo-Position einnehmen. Die Stereochemie an C-3 beweist die exo-Addition von (1) an Norbornadien.

(12)

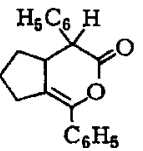

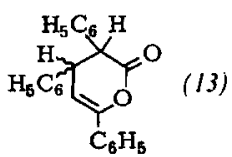

Cyclopenten ergibt mit (1) in Tetrachlormethan nach 10 d bei $100^{\circ} \mathrm{C}$ im Autoklaven nur noch $4 \%$ des Enollactons (12). Immerhin mit 24\% Ausbeute entstehen die beiden diastereomeren Dihydrotriphenyl- $\alpha$-pyrone (13), wenn (1) und Styrol in Tetrachlorethen $24 \mathrm{~h}$ auf $121^{\circ} \mathrm{C}$ erhitzt werden. Den Strukturbeweis erbrachte hier der Vergleich mit den authentischen ${ }^{[3]}$ Substanzen (13). Somit verläuft die Addition von (1) an Styrol ebenso wie jene an 1-Diethylaminopropin $^{11}$ regioselektiv.

Eingegangen am 25. November 1980 [Z 812]

[1] W. Steglich. E. Buschmann. G. Gansen, L. Wilschowitz, Synthesis 1977 ,

[2] M. Chrisll, H.-J. Lüddeke, A. Nagyrevi-Neppel, G. Freitag, Chem. Ber. 110, 3745 (1977); M. Christl, Angew. Chem. 93, 515 (1981); Angew. Chem. Int. Ed. Engl. 20, 529 (1981).

[19] S. Avery, G. C. Jongensen, J. Am. Chem. Soc, 52, 3628 (1930); R. B. Meyer. C. R. Hauser, J. Org. Chem. 27, 1067 (1962).
Valenzisomerisierung eines

Imino(methylen)phosphorans zu einem

1,2 $2 \lambda^{3}$-Azaphosphiridin:

Eine neuartige Umlagerung im Phosphorsystem ${ }^{[* *}$

Von Edgar Niecke, Anette Seyer und

Dirk-Andreas Wildbredi $t^{* 1}$

Professor Marianne Baudler zum 60. Geburtstag gewidmet

Die Phosphorverbindungen vom Ylid- $(A)^{[1]}$ und vom Dreiring-Typ $(B)^{[2]}$ haben in jüngster Zeit als wertvolle Synthesebausteine sowie aufgrund ihrer ungewöhnlichen Bindungsverhältnisse starke Beachtung gefunden.

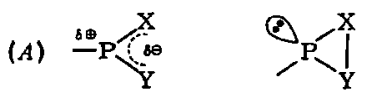

Nachdem wir kürzlich beide Verbindungsklassen aus isoelektronischen Vorstufen erzeugen konnten ${ }^{[3]}$, berichten wir hier über eine Valenzisomerisierung eines Imino(methylen)phosphorans $(A) \mathrm{zu}$ einem $1,2 \lambda^{3}$-Azaphosphiridin (B), $\mathrm{X}=\mathrm{N}-\mathrm{Y}=\mathrm{C}<{ }^{[8]}$.

Diisopropylamino(tert-butylimino)phosphan (1) reagiert mit 1-Diazo-2,2-dimethylpropan (2) zum kristallinen $[2+3]$-Cycloaddukt (3). Die bei $40^{\circ} \mathrm{C}$ einsetzende $\mathrm{N}_{2}$-Eliminierung des $\lambda^{3}$-Triazaphospholins (3) führt zum Imino(methylen)phosphoran (4), das durch Blitzdestillation in reiner Form zugănglich ist.

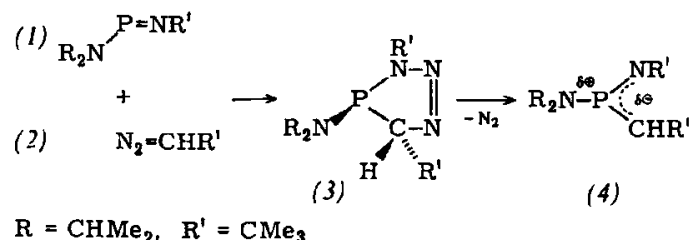

Nebenprodukt der Reaktion ist das Cycloaddukt (6), das auch aus gealterten Proben von (4) isoliert werden kann. Die bisher bei kinetisch labilen Imino(methylen)phosphoranen beobachtete Bildung von Diphosphetanen $(5)^{[4]}$ findet hier nicht statt; aufgrund sterischer Effekte ist die $[2+2]$-Cycloaddition zum Diazadiphosphetidin-System (6) begünstigt.

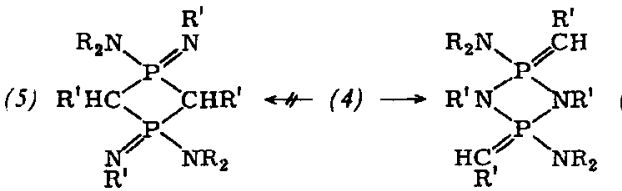

Überraschenderweise führte die thermisch induzierte Ringöffnung von ( 6 ) nicht zum erwarteten Cycloreversionsprodukt (4), sondern zu einer thermostabilen Verbindung, die sich als das zu (4) isomere, unbekannte $\lambda^{3}$-Azaphosphiridin (7) erwies. Der Nachweis für die Valenzisomerisierung $(4) \rightarrow$ (7) gelang ubber ${ }^{31} \mathrm{P}-\mathrm{NMR}$-kontrollierte Tem-

[*] Prof. Dr. E. Niecke, cand. d. L. A. Seyer, Dipl.-Chem. D.-A. Wildbredt Fakultăt für Chemie der Universităt Postfach 8640 , D-4800 Bielefeld

[**] 20. Mitteilung über Phosphazene der Koordinationszahl 2 und 3. Diese Arbeit wurde vom Fonds der Chemischen Industrie unterstatzt. - 19. Mitteilung: E. Niecke, M. Engelmann. H. Zorn, B. Krebs, G. Henkel, Angew. Chem. 92, 738 (1980); Angew. Chem. Int. Ed. Engl. 19, 709 (1980). 